\title{
Oscillation stability enhancement using multi-objective swarm based technique for SMIB system
}

\author{
N. A. M. Kamari ${ }^{1}$, I. Musirin ${ }^{2}$, M. K. M. Zamani ${ }^{3}$, S. A. Halim ${ }^{4}$ \\ ${ }^{1,4}$ Centre for Integrated Systems Engineering and Advanced Technologies (INTEGRA), \\ Faculty of Engineering and Built Environment, National University of Malaysia, Malaysia \\ ${ }^{1,4}$ Electrical and Electronic Engineering Program, Faculty of Engineering and Built Environment, \\ National University of Malaysia, Malaysia \\ ${ }^{2,3}$ Faculty of Electrical Engineering, Universiti Teknologi Mara, Malaysia
}

\begin{tabular}{l}
\hline Article Info \\
\hline Article history: \\
Received Feb 10, 2019 \\
Revised May 12, 2019 \\
Accepted May 26, 2019 \\
\hline
\end{tabular}

\section{Keywords:}

Angle stability

Damping ratio

Multi-objective

Particle swarm optimization

PID controller

\begin{abstract}
This paper discussed the impact of multi-objective function in a swarm-based optimization approach for modulate proportional-integral-derivative (PID) controller parameters of a static var compensator (SVC) in a single machine infinite bus (SMIB) system. SVC is a Flexible Alternating Current Transmission Systems (FACTS) devices which often used to increase the damping of the synchronous generator. In this paper, three parameters of PID controller: proportional gain, $\mathrm{K}_{\mathrm{P}}$, interval gain, $\mathrm{K}_{\mathrm{I}}$ and derivative gain, $\mathrm{K}_{\mathrm{D}}$ are tuned with particle swarm optimization (PSO) approach. One multi-objective function (MO) that derived from the consolidation of maximum damping factor, $\sigma_{\max }$ and minimum damping ratio, $\xi_{\min }$ is proposed to elevate the damping capability of the systems. Validation with respect to speed response and eigenvalues verification proved that the proposed MO is more competent than single objective function.
\end{abstract}

Copyright $@ 2019$ Institute of Advanced Engineering and Science. All rights reserved.

\section{Corresponding Author:}

Nor Azwan Mohamed Kamari,

Electrical and Electronic Engineering Program,

Faculty of Engineering and Built Environment,

National University of Malaysia, Malaysia.

Email: azwank@ukm.edu.my

\section{INTRODUCTION}

With the increase of the population around the world, electrical energy management becomes very important, especially in the effort to enhance the stability of the power system [1]. From the perspective of angle stability, one of the best ways to dampen the swing and improve stability is the excitation control using Flexible Alternating Current Transmission Systems (FACTS) devices. Static Var Compensator (SVC) is one of the widely used FACTS devices for power system applications. In addition to being used to control reactive power and supporting bus voltage, SVC is also capable of enhancing angle stability in the system $[2,3]$. Over the past few decades, there are various techniques that have been proposed for controlling SVC to improve the damping of synchronous engines. Among the methods proposed are proportional-integral (PI) controllers [4, 5], proportional-integral-derivative (PID) controllers [6-9] and lead lag (LL) controllers $[10,11]$. In this paper, a combination of SVC with PID controller (SVC-PID) has been chosen to improve oscillation stability in power system.

With the improvement of AI technology, optimization techniques have become an option in managing control parameters and solving other issues in power system stability. Among them are Evolutionary Programming (EP) [12-14] and Artificial Immune System (AIS) [15-17]. Both EP and AIS share almost identical features, whereas EP mimicking biological evolutionary processes, AIS works on the principle of human immune system. Although both algorithms are concise and do not use complex process 
streams, EP and AIS are not capable to provide optimal solutions and take longer computation time. In this paper, PSO has been proposed to implement the optimization process. PSO work with a simple optimization concept, resulting an easy implementation, manage to provide best optimal solutions and consumed shorter time to converge.

In searching for the optimal results, the selection of indicator used as the objective function in the optimization process is one of the important criteria. The indicator should be sensitive enough to evaluate the system, so that the optimal value of parameters can be optimized. One index is chosen as an objective function for conventional optimization techniques. Although it is successfully enhanced the damping capability, single index only improves certain property in eigenvalues. From eigenvalues point of view, usage of minimum damping ratio, $\xi_{\min }$ as objective function can shift eigenvalue region towards the real axis of phase plan by the decreasing value of imaginary part of the eigenvalue $\omega[18,19]$. Meanwhile, maximum damping factor, $\sigma_{\max }$ shows better result compared to $\xi_{\min }$ in capability of pushing the set of eigenvalues further left of the imaginary $(j \omega)$ axis of the phase plan $[20,21]$. To encompass both advantages, one multiobjective function (MO) is proposed.

This paper presents the impact of MO in PSO based angle stability improvement technique for SMIB system. The study involved an effective approach from objective function point of view, to determine the parameters of SVC damping controller in solving angle stability problems. PID controller has been chosen for the damping controller attached to the SVC system and its fixed-gains are determined using PSO optimization technique. The goal is to stabilize the system in minimum time. Along with designating MO, previously used objective functions; $\xi_{\min }$ and $\sigma_{\max }$ are selected for comparison purpose.

\section{SINGLE MACHINE CONNECTED TO AN INFINITE BUS (SMIB) SYSTEM}

Single machine connected to an infinite bus (SMIB) system is an equivalent system with one synchronous machine connected to one big bus with an infinite load. To enhance the damping capability, SMIB is equipped with a flexible AC transmission (FACTS) device called static var compensator (SVC). Widely used nowadays, SVC has very fast control response time, capable to reduce the need for maintenance of the absence of rotating parts and manage to perform individual phase control [2]. In this paper, PID controller is installed together with SVC to attempt better damping performance.

Figure 1 shows the Phillips-Heffron block diagram model for the SMIB system that equipped with SVC and PID controller (SVC-PID) [6]. SVC-PID can be segregated to SVC and PID element. SVC element involve of circuit constant, $K_{V}$ and time constant, $T_{V}$. The PID element is obtained from proportional gain, $K_{P}$, integral gain, $K_{I}$ and derivative gain, $K_{D}$ of PID controller. Before carrying out $\Delta \sigma$ to SMIB system, the value of SVC-PID have to be limited in specified upper and lower limits.

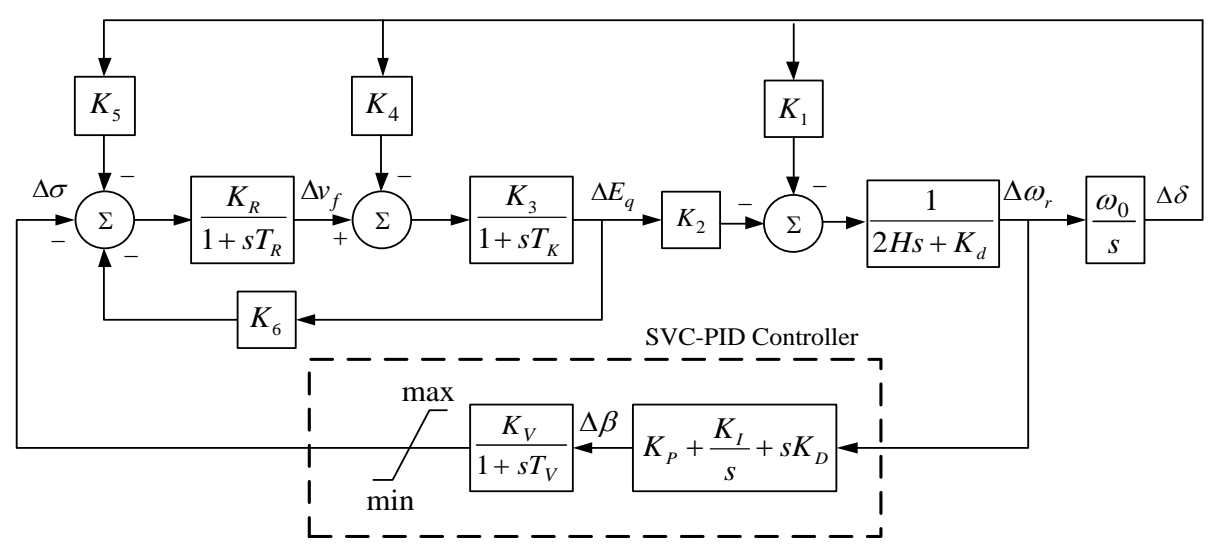

Figure 1. Phillips-Heffron block diagram model of the SMIB system with SVC-PID controller

Here, $H$ is the inertia constant, $K_{d}$ is the damping torque coefficient, $T_{R}$ and $K_{R}$ are the time constant and circuit constant of the exciter oscillation system, respectively. The value of $\omega_{0}$ is equal to $2 \pi f_{0} . K_{l}, K_{2}, K_{3}$, $T_{3}, K_{4}, K_{5}$ and $K_{6}$ are constants associated with the operating real and reactive load, impedance ratio, electrical torque, excitation level, rotor speed and rotor angle in the generator. Detail calculation of the system can be found in [4]. 


\section{MATHEMATICAL MODELLING FOR SMIB SYSTEM WITH SVC-PID}

Based on the Phillips-Heffron model of the SMIB system equipped with SVC-PID as shown in Figure 1, the following equations are applied:

$$
\begin{aligned}
& \frac{\Delta \omega}{\Delta t}=\frac{\Delta T_{m}-K_{1} \Delta \delta-K_{d} \Delta \omega_{r}-K_{2} \Delta E_{q}}{2 H} \\
& \frac{\Delta \delta}{\Delta t}=\omega_{0} \Delta \omega_{r} \\
& \frac{\Delta E_{q}}{\Delta t}=-\frac{K_{3} K_{4} \Delta \delta+\Delta E_{q}-K_{3} \Delta v_{f}}{T_{K}} \\
& \frac{\Delta v_{f}}{\Delta t}=-\frac{K_{R} K_{5} \Delta \delta+K_{R} K_{6} \Delta E_{q}+\Delta v_{f}+K_{R} \Delta \sigma}{T_{R}} \\
& \frac{\Delta \sigma}{\Delta t}=\frac{K_{V} \Delta \beta-\Delta \sigma}{T_{V}} \\
& \frac{\Delta \omega}{\Delta t}=\left(\frac{K_{D} K_{d}}{4 H^{2}}-\frac{K_{P}}{2 H}\right)\left(K_{1} \Delta \delta+K_{d} \Delta \omega_{r}+K_{2} \Delta E_{q}\right)+\left(K_{1}-\frac{K_{D} K_{1} \omega_{0}}{2 H}\right) \Delta \omega_{r} \\
& +\frac{K_{D} K_{2}}{2 H T_{K}}\left(K_{3} K_{4} \Delta \delta+\Delta E_{q}-K_{3} \Delta v_{f}\right)
\end{aligned}
$$

As shown in (1)-(6) can be encompassed into a matrix form as followed:

$$
\stackrel{\bullet}{X_{S V C}}=A_{S V C} \cdot X_{S V C}+B_{S V C} \cdot U
$$

where

$$
X_{S V C}=\left[\begin{array}{llllll}
\Delta \omega_{r} & \Delta \delta & \Delta E_{q} & \Delta v_{f} & \Delta \sigma & \Delta \beta
\end{array}\right]^{T}
$$

Comprehensive calculation of the system in Figure 1 can be found in [4]. Parameter values for the generator, transmission line, exciter and SVC controllers of the SMIB system are selected based on parameters in [6].

\section{MULTI-OBJECTIVE FUNCTIONS FOR ANGLE STABILITY IMPROVEMENT}

To elevate the damping capability of power systems, one multi-objective function (MO) derived based on combination of minimum damping ratio, $\xi_{\min }$ and maximum damping factor, $\sigma_{\max }$ is proposed. The $\xi_{\text {min }}$ is an indicator that gives the minimum value among the damping ratio of every eigenvalues in the SMIB system, based on the real and imaginary part of every eigenvalues at the loading condition. With the

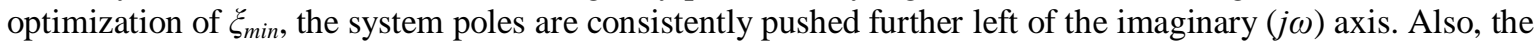
decreasing value of imaginary part of the eigenvalue $\omega$ will shift eigenvalue region towards the real axis. Effects of these combinations can be shown as a wedge-shaped sector on the phase plan [18]. The regions of eigenvalues on the phase plan, before and after optimization process are showed in Figure 2(a).

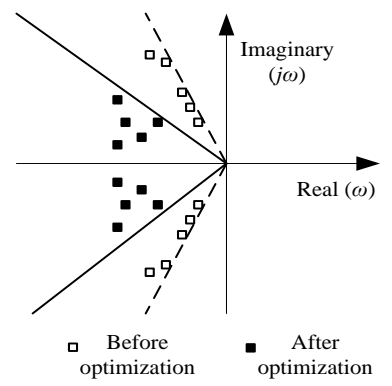

(a) with and without $\xi_{\min }$

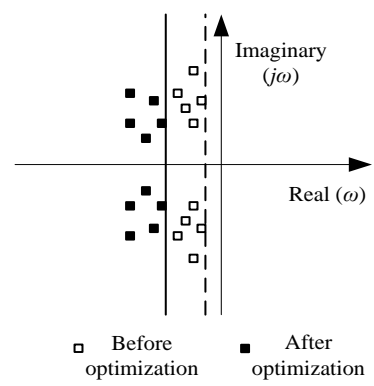

(b) with and without $\sigma_{\max }$

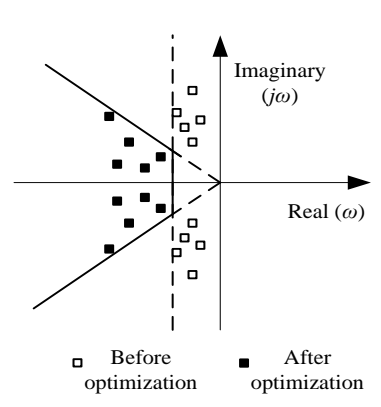

(c) with and without $\mathrm{MO}$

Figure 2. Comparison of eigenvalue areas on the phase plan 
The $\sigma_{\max }$ calculates the maximum value of damping factor $\sigma$ of every eigenvalue in the electromechanical modes of oscillation. The optimization of $\sigma_{\max }$ will result the system poles to be shifted further left of the $j \omega$ axis [20]. The regions of eigenvalues on the phase plan; before and after optimization process are showed in Figure 2(b). By implementing the proposed MO, area of eigenvalues will shift further left of the imaginary axis and at the same time towards real axis. The area of eigenvalues on the phase plan for comparison case between system with MO approach and without MO approach is shown in Figure 2(c). The area bounded by MO effect can be shown as a trapezoid-shaped sector on the phase plan. To combine both $\xi_{\min }$ and $\sigma_{\max }$ into one fitness equation, an objective function is designated as follows:

$$
J_{M O}=\alpha_{1} \cdot \xi_{\min }+\alpha_{2} \cdot \sigma_{\max }=\alpha_{1} \cdot \min \left(-\sigma_{i} / \sqrt{\sigma_{i}^{2}+\omega_{i}^{2}}\right)+\alpha_{2} \cdot \max \left(\sigma_{\max }\right), 0 \leq \alpha_{1}, \alpha_{2} \leq 1
$$

$\alpha_{1}$ and $\alpha_{2}$ are constants; attached to $\xi_{\min }$ and $\sigma_{\max }$, respectively to tune the percentage of both indicators. $\sigma_{i}$ and $\omega_{i}$ are respectively the real and imaginary part of the $i^{\text {th }}$ eigenvalue. The combination of the objective function can be formulated as:

$\operatorname{Maximize}\left(J_{m o}\right)$

$J_{m o}$ refers to multi-objective function (MO). Following limitations must be complied for SVC-PID system:

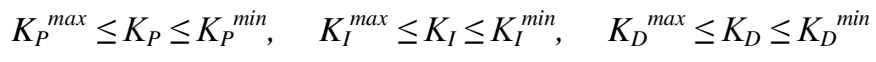

To estimate the maximum value of $J_{m o}$, the following process was implemented:

a) Calculate eigenvalues using (7)

b) Evaluate $J_{m o}$ using (9).

c) Vary the value of selected parameters and repeat step (i) and (ii) until the difference between the maximum and minimum value of objective function, $J_{\max }$ and $J_{\min }$, respectively reaches 0.001 or number of iterations reach 100 .

\section{PARTICLE SWARM OPTIMIZATION (PSO) TECHNIQUE}

Particle Swarm Optimization (PSO) technique is developed based on the movement of flock of birds flocking and school of fish in searching for food. PSO tries to adopt the principle of individual intelligence as well as the influence of collective behavior of the herd. This algorithm involved initialization, velocity and positions update, fitness calculation and best position update. The flow chart which represents PSO algorithm is illustrated in Figure 3. Completed descriptions of PSO algorithm process can be found in [16].

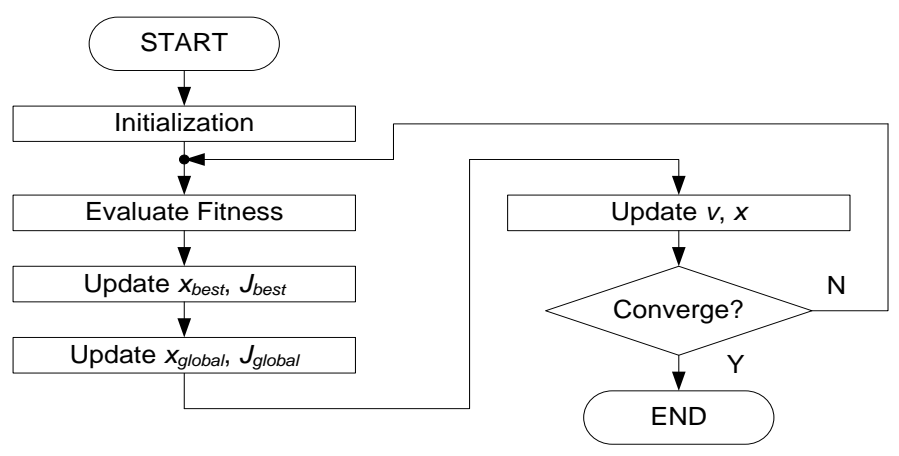

Figure 3. Flowchart for the particle swarm optimization (PSO) algorithm

Here, $K_{P}, K_{I}$ and $K_{D}$ are optimized by PSO method. The parameters involved in the proposed approach is tabulated in Table 1.

Table 1. The Parameters for PSO Algorithm

List of Parameters $\quad c_{1}=c_{2}=0.5, \omega_{\max }=0.09, \omega_{\min }=0.04$

Indonesian J Elec Eng \& Comp Sci, Vol. 16, No. 2, November 2019 : 631-639 


\section{RESULTS AND ANALYSIS}

In this paper, two cases were implemented to search the most suitable ratio of $\xi_{\min }$ and $\sigma_{\max }$ that compress in one multi-objective function (MO). Both cases were conducted using single machine connected to an infinite bus (SMIB) system in MATLAB environment. The simulation used SVC-PID system with Particle Swarm Optimization (PSO) as the computational intelligence technique. All values of $K_{P}, K_{I}$ and $K_{D}$ parameters are randomly selected. The simulations are simulated in two different loading conditions as tabulated in Table 2. For loading conditions which are less or more than the values simulated in Table 2, they are considered to produce the similar results.

Table 2. Three Different Loading Conditions for Case 1 and Case 2

\begin{tabular}{cccc}
\hline & Case & 1 & 2 \\
\hline Loading & Active power, P (p.u.) & 0.35 & 0.75 \\
condition & Reactive power, Q (p.u.) & -0.2 & 0.5 \\
\hline
\end{tabular}

$\xi_{\min }$ and $\sigma_{\max }$ have different value characteristics. The value of $\xi_{\min }$ is in the range of [0,1]. On the contrary, there are large possibility of value for $\sigma_{\max }$ (from $-\infty$ to $+\infty$ ). To acquire the benefits of both indicators, the merger of the two indexes is essential. For this to happen, the $\sigma_{\max }$ is reduced to maximum $10 \%$ of actual value, so one index will not monopolize other index in the new indicators.

In searching for the most suitable value of $\xi_{\min }$ and $\sigma_{\max }$, twelve different MO combinations have been introduced based on the (9). Comparison of constants $\alpha_{1}$ and $\alpha_{2}$ for all twelve different MO combinations for all cases are tabulated in Table 3.

Table 3. Comparisons of Ratio Constants $\alpha_{1}$ and $\alpha_{2}$ for all Twelve MO Combinations

\begin{tabular}{cccccc}
\hline \multirow{2}{*}{ MO Combinations } & \multicolumn{2}{c}{ Ratio constants } & \multirow{2}{*}{ MO Combinations } & \multicolumn{2}{c}{ Ratio constants } \\
& $\alpha_{1}$ & $\alpha_{2}$ & MO-6 & 1 & 0.06 \\
Zt & 1 & 0 & MO-7 & 1 & 0.07 \\
MO-1 & 1 & 0.01 & MO-8 & 1 & 0.08 \\
MO-2 & 1 & 0.02 & MO-9 & 1 & 0.09 \\
MO-3 & 1 & 0.03 & MO-10 & 1 & 0.10 \\
MO-4 & 1 & 0.04 & Sg & 0 & 0.10 \\
MO-5 & 1 & 0.05 & &
\end{tabular}

To analyze the results for all three cases, all responses are divided into two groups: Group 1 and Group 2. Group 1 consist of result for Zt, MO-1, MO-2, MO-3, MO-4, MO-5 and MO-6, while Group 2 cover result for MO-6, MO-7, MO-8, MO-9, MO-10 and Sg. Figure 4(a) and 4(b) show the response of speed deviation of Group 1 and Group 2 for Case 1, respectively.

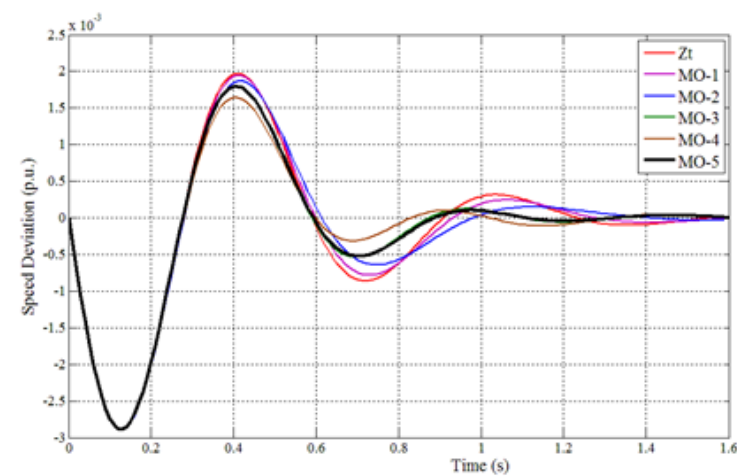

(a) Group 1 (MO-1 MO-5 and Zt)

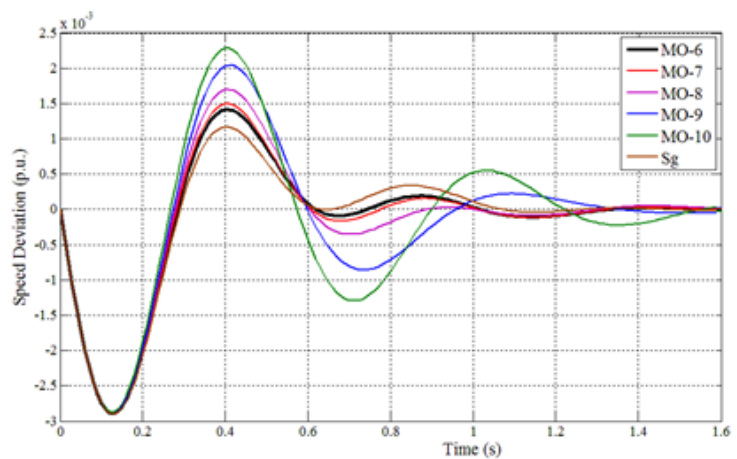

(b) Group 2 (MO-6 MO-10 and $\mathrm{Sg}$ )

Figure 4. Speed response in various combination for Case 1

From Figure 4(a) and 4(b), it shows that all combinations are completely damped within 1.6 seconds of total computation time, which indicate that all twelve approaches demonstrate stable condition. From the 
speed deviation response, the oscillation patterns are decreased from MO-1 to MO-6 approaches, gradually, and increased again from MO-7 to MO-10. From the comparison of responses, it is discovered that MO-6 gives the fastest damping responses and less oscillation, followed by MO-7. Zt approach shows almost the same result with MO-1. On the other hand, $\mathrm{Sg}$ gives the biggest oscillation rate, which makes it the worst among all the twelve combinations.

The regions of eigenvalues location in complex s-plane for Group 1 and Group 2 respectively are shown in Figure 5(a) and 5(b). Regarding eigenvalues, the one located at the left-hand side of s-plane, which justifies that all the twelve system approaches are stable. Among those twelve combinations, the eigenvalues' region based on MO-6 approach is located far to the left side of s-plane and closer to the real-axis. This result revealed that the MO-6 combination is the most prominent approach from the rest. Meanwhile, $\mathrm{Zt}$ and $\mathrm{Sg}$ approaches are among the worst combinations. In spite of positioned at the left-hand side area, the region of eigenvalues for $\mathrm{Zt}$ is at the nearest position to the right-hand side of s-plane compared to the other eleven combinations. For $\mathrm{Sg}$, the eigenvalues distributions are located further away from the real-axis.

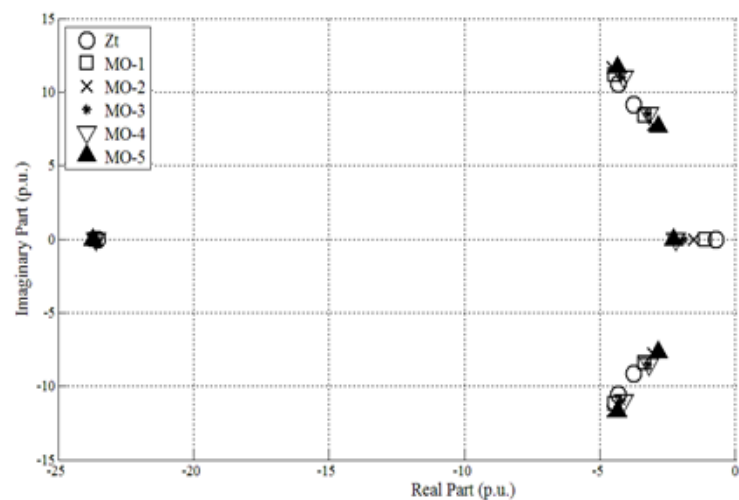

(a) Group 1 (MO-1 MO-5 and Zt)

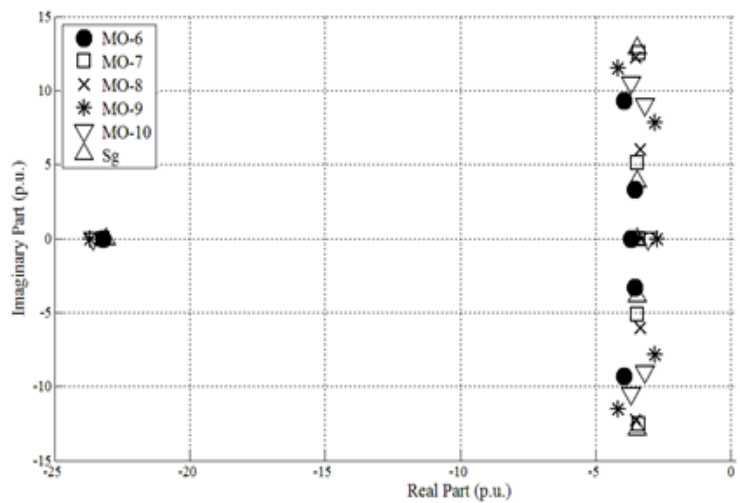

(b) Group 2 (MO-6 MO-10 and $\mathrm{Sg}$ )

Figure 5. Location of eigenvalues for Case 1

The comparisons of SVC-PID parameters based on twelve multi-objective (MO) combinations for Case 1 tabulated in Table 4. The optimized values of $K_{P}$ for all twelve combinations are in narrow range, between 0.5039 and 0.5693 . The same pattern of results is also obtained for $K_{I}(-0.0414 \sim-0.0005)$ and $K_{D}$ (0.1461 0.5611). This shows that all twelve combinations tuned the SMIB systems almost in the same manner, with slightly different value. This also indicates that the tuning process of damping parameters is very dependent on the selection of the objective function. In this critical process, only the best combination will calculate the most suitable SVC-PID parameters and gives the most improvement in stability condition of SMIB system.

Table 4. Comparisons of SVC-PID Parameters Based on MO Combinations for Case 1

\begin{tabular}{ccccccc}
\hline \multirow{2}{*}{ MO Combinations } & \multicolumn{3}{c}{ SVC-PID Parameters } & \multirow{2}{*}{ MO Combinations } & \multicolumn{2}{c}{ SVC-PID Parameters } \\
& $K_{P}$ & $K_{I}$ & $K_{D}$ & & $K_{P}$ & $K_{D}$ \\
\hline Zt & 0.5106 & -0.0212 & 0.1461 & MO-6 & 0.5282 & -0.0384 \\
MO-1 & 0.5362 & -0.0174 & 0.2129 & MO-7 & 0.5192 & -0.0332 \\
MO-2 & 0.5693 & -0.0183 & 0.2721 & MO-8 & 0.5262 & -0.0210 \\
MO-3 & 0.5255 & -0.0064 & 0.3528 & MO-9 & 0.5510 & -0.0070 \\
MO-4 & 0.5242 & -0.0046 & 0.4031 & MO-10 & 0.5039 & -0.0005 \\
MO-5 & 0.5649 & -0.0111 & 0.3877 & Sg & 0.5315 & -0.0414 \\
\hline
\end{tabular}

The speed deviation response of Group 1 and Group 2 for Case 2 are shown in Figure 6(a) and Figure 6(b), respectively. As those in the previous case, all twelve MO combinations are capable to elevate the stability condition of SMIB system. In this case, MO-3, MO-4, MO-5, MO-6 and MO-7 are the top five achievers in giving the low oscillation rate in speed deviation response. In other hand, $\mathrm{Zt}$ and $\mathrm{Sg}$ approaches are among the worst, resulting bigger oscillation compare to other combinations. This shows that the two-inone objective function is very convincing in giving better damping capability, rather than a single objective function. 


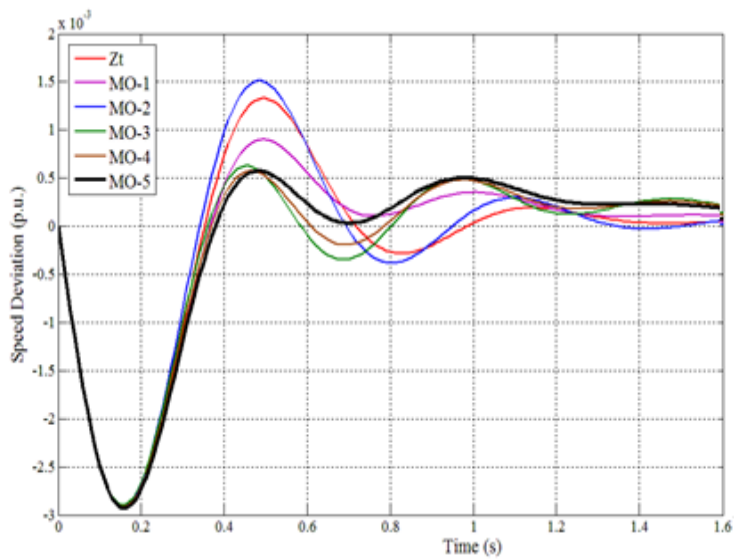

(a) Group 1 (MO-1 MO-5 and Zt)

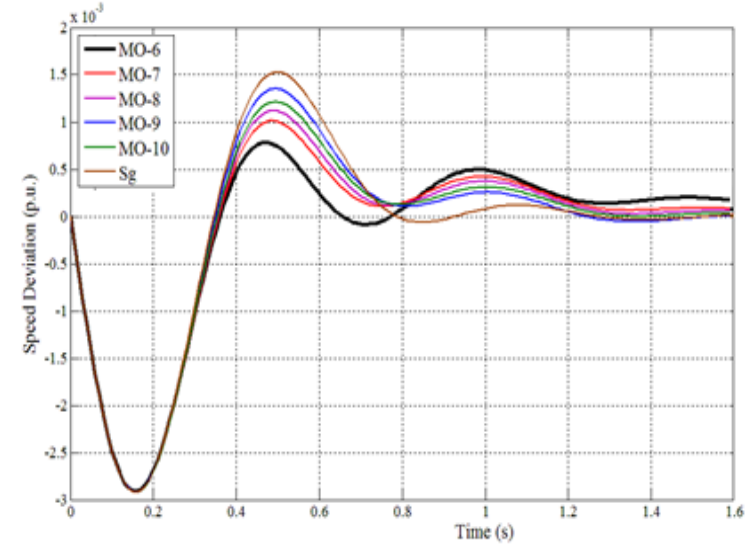

(b) Group 2 (MO-6 MO-10 and Sg)

Figure 6. Speed response in various combination for Case 2

The regions of eigenvalues location in complex s-plane for Group 1 and Group 2 for Case 2 are shown in Figure 7(a) and 7(b). In this case, the regions of eigenvalues for all twelve combinations are positioned at the left-hand side of s-plane. Among those twelve, MO-6 is succeeded to shift its eigenvalues region to the farthest on the left side of s-plane and most close to real-axis. This justifies that the combination of MO-6 is the most suitable approach for Case 2. On the other hand, the approach of Sg gives good result by positioning the eigenvalues coordinate to the far left of s-plane. But, as two of the eigenvalues are situated at the farthest from real-axis, it is making Sg not so efficient to improve the stability condition of the system. Meanwhile, one of eigenvalues of $\mathrm{Zt}$ method is positioned at the nearest to origin but at the right-hand side of s-plane, resulting $\mathrm{Zt}$ as the worst objective function approach.

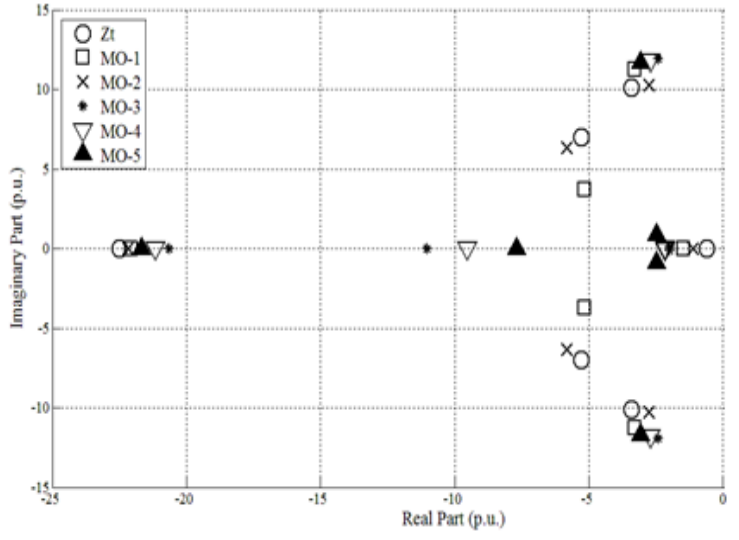

(a) Group 1 (MO-1 MO-5 and $\mathrm{Zt}$ )

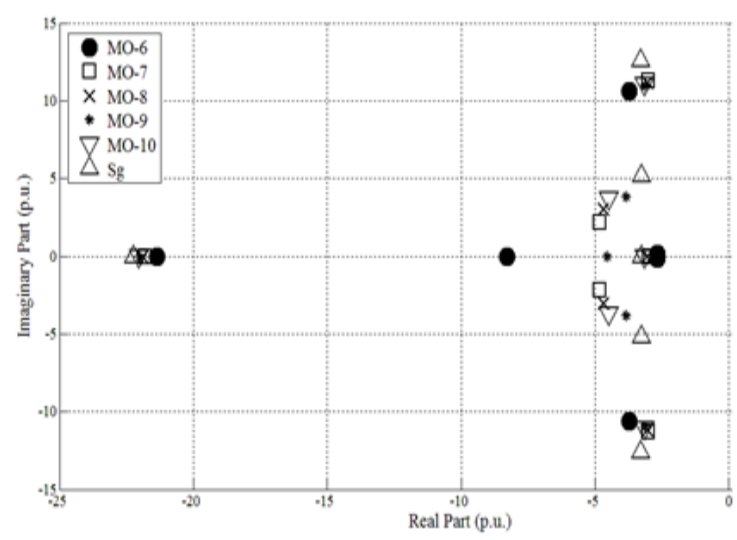

(b) Group 2 (MO-6 MO-10 and Sg)

Figure 7. Location of eigenvalues for Case 2

Table 5 tabulates the comparisons of SVC-PID parameters based on MO combinations for Case 2.

Table 5. Comparisons of SVC-PID Parameters Based on MO Combinations for Case 2

\begin{tabular}{ccccccc}
\hline \multirow{2}{*}{ MO Combinations } & \multicolumn{3}{c}{ SVC-PID Parameters } & \multirow{2}{*}{ MO Combinations } & \multicolumn{3}{c}{ SVC-PID Parameters } \\
& $K_{P}$ & $K_{I}$ & $K_{D}$ & MO-6 & 0.5232 & -0.3028 \\
\hline Zt & 0.6281 & -0.3887 & 0.0815 & MO-7 & 0.5773 & -0.2021 \\
MO-1 & 0.6124 & -0.2900 & 0.1276 & 0.1285 & 0.1775 \\
MO-2 & 0.5732 & -0.1988 & 0.1458 & MO-8 & 0.5870 & -0.1744 \\
MO-3 & 0.4527 & -0.4492 & 0.0932 & MO-9 & 0.6021 & -0.1191 \\
MO-4 & 0.5130 & -0.4235 & 0.0988 & MO-10 & 0.6025 & -0.1535 \\
MO-5 & 0.5822 & -0.3457 & 0.1193 & Sg & 0.6235 & -0.1077 \\
\hline
\end{tabular}


Two cases presented have proved that the combination of maximum damping ratio $\xi_{\min }$ and maximum damping factor $\sigma_{\max }$ as multi-objective function is succeeded to enhance the optimization capability to become more accurate in tuning SVC-PID parameters. Almost all the combinations which have been presented demonstrate better damping results as compared to conventional single objective functions such as $\xi_{\min }$ and $\sigma_{\max }$. Among those ten combinations, the MO-6 $\left(\alpha_{1}=1, \alpha_{2}=0.06\right)$ approach shows the most prominent results based on two responses; speed deviation and eigenvalues region. Using this approach, PSO optimization technique constantly identify the optimal values of $K_{P}, K_{I}$ and $K_{D}$ to decrease the oscillation rate of speed deviation. In complex s-plane result, the MO-6 approach is also capable to shift the eigenvalues position to the most left of left-hand side of imaginary-axis and to the nearest to real-axis.

\section{CONCLUSION}

From this paper, it can be concluded that introduction of multi-objective (MO) manage to elevate the stability quality of power system. The application of minimum damping ratio $\xi_{\min }$ as objective function of an optimization process will shift the eigenvalues' region further to left side of complex plane, while the usage of maximum damping factor $\sigma_{\max }$ will bring eigenvalues position towards the real axis of s-plane. Merger of $\xi_{\min }$ and $\sigma_{\max }$ index in the certain ratio as objective function will bring both advantages into the selected optimization process. From Cases 1 and 2, both results indicate that combination of $\xi_{\min }$ and $\sigma_{\max }$ approach at $\alpha_{1}=1$ and $\alpha_{2}=0.06$ elevate the performance of PSO computation technique, given the best damping capability of SVC-PID controller among all approaches.

\section{ACKNOWLEDGEMENTS}

The authors would like to acknowledge Centre For Research and Instrumentation Management (CRIM), UKM, Bangi, Selangor, Malaysia for the financial support of this research. This research is supported by Geran Galakan Penyelidik Muda (GGPM) with project code: GGPM-2018-055.

\section{REFERENCES}

[1] M. A. Hannan, et al., "Artificial Intelligent Based Damping Controller Optimization for the Multi-Machine Power System: A Review," IEEE Access, vol. 6, pp. 39574-39594, Jul 2018.

[2] M. A. Hannan, et al., "Artificial Intelligent Based Damping Controller Optimization for the Multi-Machine Power System: A Review," IEEE Access, vol. 6, pp. 39574-39594, Jul 2018.

[3] S. C. M. Nasir, et al., "Multistage Artificial Immune System for Static VAR Compensator Planning," Indonesian Journal of Electrical Engineering and Computer Science, vol. 14, pp. 346-352, Apr 2019.

[4] N. A. M. Kamari, et al., "Optimal Tuning of SVC-PI Controller using Whale Optimization Algorithm for Angle Stability Improvement," Indonesian Journal of Electrical Engineering and Computer Science, vol. 12, pp. 612-619, Nov 2018.

[5] S. S. Khorramabadi and A. Bakhshai, "Critic-Based Self-Tuning PI Structure for Active and Reactive Power Control of SVCs in Microgrid Systems," IEEE Transactions on Smart Grid, vol. 6, pp. 92-103, Sep 2014.

[6] N. A. M. Kamari, et al., "IPSO based SVC-PID for Angle Stability Enhancement," International Journal of Simulation: Systems, Science and Technology, vol. 17, pp. 20.1-20.7, Jan 2017.

[7] T. Chaiyatham and I. Ngamroo, "Improvement of Power System Transient Stability by PV Farm with Fuzzy Gain Scheduling of PID Controller," IEEE System Journal, vol. 11, pp. 1684-1691, Sep 2014.

[8] A. Banerjee, et al., "Seeker Optimized SVC-PID Controller for Reactive Power Control of An Isolated Hybrid Power System," Energy Systems, vol. 9, pp. 1-31, Aug 2018.

[9] P. K. Ray, et al., "Improvement of Stability in Solar Energy Based Power System Using Hybrid PSO-GS Based Optimal SVC Damping Controller," Energy Procedia, vol. 109, pp. 130-137, Mar 2017.

[10] N. A. M. Kamari, et al., "PSS Based Angle Stability Improvement using Whale Optimization Approach," Indonesian Journal of Electrical Engineering and Computer Science, vol. 8, pp. 382-390, Nov 2017.

[11] M. Shafiullah, et al., "Designing Lead-Lag PSS Employing Backtracking Search Algorithm to Improve Power System Damping," 2017 Nineth IEEE-GCC Conference and Exhibition (GCCCE), pp. 1-9, Aug 2018.

[12] S. A. S. Mustaffa, et al., "Load Management for Voltage Stability Control in Multi-Area Power System," $6^{\text {th }}$ IEEE International Conference on Control System, Computing and Engineering (ICCSCE), pp. 502-506, Nov 2016.

[13] N. A. Salim, et al., "Optimal Allocation of FACTS Device to Improve Voltage Profile and Power Loss Using Evolutionary Programming Technique," 2016 IEEE Region 10 Conference (TENCON), pp. 1208-1215, Nov 2016.

[14] S. Chansareewittaya, "Enhancing Ration of TTC Per Fuel Cost Using Evolutionary Programming with UPFC," $20185^{\text {th }}$ International Conference on Business and Industrial Research (ICBIR), pp. 304-308, May 2018.

[15] R. Alonso, et al., "Short Term Load Forecast Method Using Artificial Neural Network with Artificial Immune Systems," 2017 IEEE URUCON, pp. 1-4, Oct 2017.

[16] S. Saravanan, et al., "Estimation of India's Energy Demand Using Artificial Immune System," 2017 International Conference on Intelligent Computing and Control Systems (ICICCS), pp. 1150-1154, Jun 2017. 
[17] F. R. Alonso, et al., "Artificial Immune Systems Optimization Approach for Multi-Objective Distribution System Reconfiguration," IEEE Transactions on Power Systems, vol. 30, pp. 840-847, Mar 2015.

[18] T. Tarif, et al., "Optimal PMU Placement for Small-Signal Stability Assessment using Genetic Algorithm,” 2018 International Conference on Electrical Sciences and Technologies in Maghreb (CISTEM), pp. 1-6, Jan 2019.

[19] R. T. Bernardo, et al., "An Improved LMI-Based Approach for the Design of Power System Damping Controllers," IFAC-PapersOnLine, vol. 51, pp. 287-292, 2018.

[20] J. Alipoor, et al., "Power System Stabilization using Virtual Synchronous Generator with Alternating Moment of Inertia," IEEE Journal of Emerging and Selected Topics in Power Electronics, vol. 3, pp. 451-458, Oct 2014.

[21] N. N. Islam, et al., "An Application of Backtracking Search Algorithm in Designing Power System Stabilizers for Large Multi-Machine System,” Neurocumputing, vol. 237, pp. 175-184, May 2017. 